\title{
CANONICAL BIASSOCIATIVE GROUPOIDS
}

\author{
Biljana Janeva, Snežana Ilić, and \\ Vesna Celakoska-Jordanova
}

\begin{abstract}
In the paper Free biassociative groupoids, the variety of biassociative groupoids (i.e., groupoids satisfying the condition: every subgroupoid generated by at most two elements is a subsemigroup) is considered and free objects are constructed using a chain of partial biassociative groupoids that satisfy certain properties. The obtained free objects in this variety are not canonical. By a canonical groupoid in a variety $\mathcal{V}$ of groupoids we mean a free groupoid $(R, *)$ in $\mathcal{V}$ with a free basis $B$ such that the carrier $R$ is a subset of the absolutely free groupoid $\left(T_{B}, \cdot\right)$ with the free basis $B$ and $(t u \in R \Rightarrow t, u \in R \& t * u=t u)$. In the present paper, a canonical description of free objects in the variety of biassociative groupoids is obtained.
\end{abstract}

\section{Preliminaries}

Let $\boldsymbol{G}=(G, \cdot)$ be a groupoid and $a, b \in G$. We denote by $\langle a, b\rangle$ the subgroupoid of $\boldsymbol{G}$ generated by $a, b$ and by $\langle a\rangle$ the subgroupoid generated by $a$. Clearly, $\langle a\rangle \subseteq$ $\langle a, b\rangle$ and if $b \in\langle a\rangle$, then $\langle a, b\rangle=\langle a\rangle$; specially, $\langle a, a\rangle=\langle a\rangle$. The subgroupoids $\langle a, b\rangle$ and $\langle b, a\rangle$ are equal.

Let $a_{1}, a_{2}, \ldots, a_{n}$ be a finite sequence of elements in a groupoid $\boldsymbol{G}$. We denote by $a_{1} a_{2} \cdots a_{n}$ the product of the sequence $a_{1}, a_{2}, \ldots, a_{n}$ in $\boldsymbol{G}$ defined as follows:

i) if $n=3$, then $a_{1} a_{2} a_{3} \stackrel{\text { def }}{=} a_{1}\left(a_{2} a_{3}\right)$ and

ii) if $n \geqslant 3$, then $a_{1} a_{2} \cdots a_{n} \stackrel{\text { def }}{=} a_{1}\left(a_{2} \cdots a_{n}\right)$.

We call $a_{1} a_{2} \cdots a_{n}$ the main product of the sequence $a_{1}, a_{2}, \ldots, a_{n}$. If $n=1$ and $n=2$, then $a_{1}$ and $a_{1} a_{2}$ will also be called the main products of the sequences $a_{1}$ and $a_{1}, a_{2}$ respectively. If $c=a_{1} a_{2} \cdots a_{n}$, then we say that $c$ is presented as a main product of the sequence $a_{1}, a_{2}, \ldots, a_{n}$.

Let $\boldsymbol{G}$ be a groupoid and $A \subseteq G$. If $\boldsymbol{Q}$ is the subgroupoid of $\boldsymbol{G}$ generated by $A$, i.e., $\boldsymbol{Q}=\langle A\rangle$, then $Q=\bigcup\left\{A_{k}: k \geqslant 0\right\}$, where $A_{0}=A, A_{k+1}=A_{k} \cup A_{k} A_{k}$.

If $x \in Q$, then a hierarchy of $x$ in $Q$ is the nonnegative integer $\chi_{\boldsymbol{Q}}(x)$, defined by $\chi_{\boldsymbol{Q}}(x)=\min \left\{k \in \mathbb{N}_{0}: x \in A_{k}\right\}$, where $\mathbb{N}_{0}$ is the set of nonnegative integers.

2000 Mathematics Subject Classification: Primary 08B20; Secondary 03 C05.

Key words and phrases: Groupoid, subgroupoid generated by two elements, subsemigroup, free groupoid, canonical groupoid. 
In the sequel $B$ will be an arbitrary nonempty set whose elements are called variables. By $T_{B}$ we will denote the set of all groupoid terms over $B$ in the signature $\cdot$. The terms are denoted by $t, u, v, \ldots, x, y, \cdots \boldsymbol{T}_{B}=\left(T_{B}, \cdot\right)$ is the absolutely free groupoid with the free basis $B$, where the operation is defined by $(u, v) \mapsto u v$. The groupoid $\boldsymbol{T}_{B}$ is injective, i.e., if $x, y, v, w \in T_{B}$, then $x y=v w \Rightarrow x=v, y=w$; in other words the operation $\cdot$ is an injective mapping.

Note that $\boldsymbol{T}_{B}=\bigcup\left\{B_{k}: k \geqslant 0\right\}$, where $B_{0}=B, B_{k+1}=B_{k} \cup B_{k} B_{k}$. The hierarchy $\chi: T_{B} \rightarrow \mathbb{N}_{0}$, defined by $\chi(t)=\min \left\{k \in \mathbb{N}_{0}: t \in B_{k}\right\}$, for any $t \in T_{B}$, has the property:

$$
\chi(t u)=1+\max \{\chi(t), \chi(u)\},
$$

for all $t, u \in T_{B}$.

For any term $v \in T_{B}$ we define the length $|v|$ of $v$ and the set of subterms $P(v)$ of $v$ in the following way:

$$
|b|=1,|t u|=|t|+|u| ; P(b)=\{b\}, P(t u)=\{t u\} \cup P(t) \cup P(u),
$$

for any $b \in B$ and $t, u \in T_{B}$.

\section{Main biproducts}

Let $t, u \in T_{B}$ and $\langle t, u\rangle$ be the subgroupoid of $\boldsymbol{T}_{B}$ generated by $t, u$ :

$$
\langle t, u\rangle=\{t, u, t t, t u, u t, u u, t(t t), t(t u), t(u t), t(u u),(t t) t,(t u) t, \cdots\} .
$$

Each element $x$ of $\langle t, u\rangle$ is a product of a finite sequence of elements $x_{1}, \ldots, x_{n}$ $(n \geqslant 1)$, where each $x_{i}$ is either $t$ or $u$, i.e., $\left\{x_{1}, x_{2}, \ldots, x_{n}\right\} \subseteq\{t, u\}$. Any such product is constructed by the two generators $t, u$ and therefore we call it a binary product or shortly biproduct.

Thus, if a term $x \in T_{B}$ is an element of $\langle t, u\rangle$, then we say that $x$ has a representation as a biproduct (or shortly, $x$ is a biproduct) with the generating pair $\{t, u\}$ and denote it by $x_{\langle t, u\rangle}$. (In this case we also say that $x$ is the carrier of the biproduct $x_{\langle t, u\rangle}$.)

If $u=t$ or $u \in\langle t\rangle$, then $\langle t, u\rangle=\langle t\rangle$. In that case if $x \in\langle t\rangle$, we say again that $x$ is a biproduct with the generator $t$ and denote it by $x\langle t\rangle$. Specially, $t \in\langle t\rangle$ and $t$ has a representation as a biproduct with the generator $t: t_{\langle t\rangle}=t$. We say that $t_{\langle t\rangle}$ is a trivial biproduct of $t$. Since $t \in\langle t, u\rangle$ we have $t_{\langle t, u\rangle}=t$ and we say also that $t_{\langle t, u\rangle}$ is the trivial biproduct of $t$ in $\langle t, u\rangle$.

If $t \notin\langle u\rangle$ and $u \notin\langle t\rangle$, then no two elements of the subgroupoid $\langle t, u\rangle$ are equal, since the groupoid $\boldsymbol{T}_{B}$ is injective. Therefore:

Proposition 2.1. If $t, u, x$ are terms of $T_{B}$ and $x$ is such that $x \in\langle t, u\rangle$, $t \notin\langle u\rangle$ and $u \notin\langle t\rangle$, then $x$ has a unique representation as a biproduct with the generating pair $\{t, u\}$.

Note that a term of $T_{B}$ may have representations as biproducts with different pairs of generators.

EXAMPLE 2.1. Let $a, b$ be two distinct variables and $x$ the term $((a b) b)(a b)$.

1) $x \in\langle x\rangle$, and thus $x_{\langle x\rangle}=x$ is the biproduct of $x$ with the generator $x$. 
2) Put $t=(a b) b$ and $u=a b$. Then $x \in\langle t, u\rangle$ and $x_{\langle t, u\rangle}=t u$ is the biproduct of $x$ with the generating pair $\{t, u\}$.

3) If $u=a b$ and $v=b$, then $x \in\langle u, v\rangle$ and $x_{\langle u, v\rangle}=(u v) u$ is the biproduct of $x$ with the generating pair $\{u, v\}$.

4) $x \in\langle a, b\rangle$ and thus $x_{\langle a, b\rangle}=((a b) b)(a b)$ is the biproduct of $x$ with the generating pair $\{a, b\}$.

(Note that there is no biproduct of $x$ other than those enumerated above.)

A biproduct $x_{\langle t, u\rangle}$ of a term $x$ is said to be maximal in $\boldsymbol{T}_{B}$ if and only if for any biproduct $x_{\langle\alpha, \beta\rangle}$ of $x$, the hierarchy $\chi_{\langle\alpha, \beta\rangle}(x)$ does not exceed the hierarchy $\chi_{\langle t, u\rangle}(x)$, i.e., $\chi_{\langle\alpha, \beta\rangle}(x) \leqslant \chi_{\langle t, u\rangle}(x)$.

Proposition 2.2. Any term $x$ of $T_{B}$ has a finite number of representations as a biproduct in $\boldsymbol{T}_{B}$, i.e., $x \in T_{B}$ is the carrier of a finite number of biproducts in $\boldsymbol{T}_{B}$. Any term $x$ of $T_{B}$ is the carrier of maximal biproducts in $\boldsymbol{T}_{B}$.

Proof. The length $|x|$ of any $x \in T_{B}$ is finite, and thus the set $P(x)$ of subterms of $x$ is finite. As the generators of any biproduct of $x$ are subterms of $x$, and the set of subterms $P(x)$ of $x$ is finite, it follows that $x$ has a finite number of biproducts. The set of nonnegative integers that are hierarchies of $x$ (with respect to the pair of generators of all biproducts of $x$, including the pairs $\{t, t\}=\{t\}$ ) is finite, and thus it has the largest element. Therefore, there is the largest hierarchy of $x$, i.e., a maximal biproduct of $x$.

Note that a given term $x$ of $T_{B}$ may have more than one maximal biproducts.

EXAMPLE 2.2. Let $x=((a b) b)\left(b^{2}(a b)\right)$ (where $a, b$ are variables). Put $t=a b$ and $u=b$. Then $x_{\langle t, u\rangle}=(t u)\left(u^{2} t\right)$ and $\chi_{\langle t, u\rangle}(x)=3$. If we take $\{a, b\}$ as the generating pair, then $x_{\langle a, b\rangle}=((a b) b)\left(b^{2}(a b)\right)$ is a biproduct of $x$ and $\chi_{\langle a, b\rangle}(x)=3$. For all other biproducts $x_{\langle\alpha, \beta\rangle}$ one obtains that $\chi_{\langle\alpha, \beta\rangle}(x) \leqslant 3$. Thus, $x_{\langle t, u\rangle}$ and $x_{\langle a, b\rangle}$ are maximal biproducts of $x$.

Let $x=x_{1} x_{2} \cdots x_{m}$ be the main product of $x_{1}, x_{2}, \ldots, x_{m}$ in $\boldsymbol{T}_{B}$. If

$$
\left\{x_{1}, x_{2}, \ldots, x_{m}\right\} \subseteq\{t, u\},
$$

for some terms $t, u$ of $T_{B}$, then we call $x_{1} x_{2} \cdots x_{m}$ the main biproduct of $x$ in $\boldsymbol{T}_{B}$ with the generating pair $\{t, u\}$ and denote it by $x_{t, u}$. (If $u=t$, i.e., the generating "pair" is $\{t, t\}$, we write $x_{t}$ instead of $x_{t, t}$.)

Below we will state some properties about main biproducts.

(1) Note that any term $x$ of $T_{B}$ has at least one main biproduct - the trivial one, $x_{x}$. If $x \in T_{B} \backslash B$, then $x=\alpha \beta$ for some $\alpha, \beta \in T_{B}$, and $x_{\alpha, \beta}=\alpha \beta$ is another main biproduct of $x$ in $\boldsymbol{T}_{B}$.

(2) The hierarchy of a main biproduct $x_{1} x_{2} \cdots x_{m}$, with a generating pair $\{t, u\}$, equals $m-1$. Therefore, if two main biproducts $x_{1} x_{2} \cdots x_{m}$ and $y_{1} y_{2} \cdots y_{m+k}$ are maximal biproducts of $x$ in $\boldsymbol{T}_{B}$, then they have to satisfy $k=0$ (or the hierarchies would differ) and $x_{i}=y_{i}$, for $1 \leqslant i \leqslant m$.

Proposition 2.3. If $x \in T_{B}$ has two nontrivial main biproducts $x_{t, u}$ and $x_{v, w}$ in $\boldsymbol{T}_{B}$, then one generator of the one generating pair coincides with a generator of the other generating pair. 
Proof. Let $x_{t, u}=x_{1} x_{2} \cdots x_{m}$ and $x_{v, w}=y_{1} y_{2} \cdots y_{n}$ be two main biproducts of $x$ in $\boldsymbol{T}_{B}$. Then $x_{1} x_{2} \cdots x_{m}=y_{1} y_{2} \cdots y_{n}$ implies $x_{1}=y_{1}$. Since $x_{\nu} \in\{t, u\}$ and $y_{\lambda} \in\{v, w\}$ it follows that $x_{1}$ is either $t$ or $u$, and $y_{1}$ is either $v$ or $w$. If, for example, $x_{1}=t$ and $y_{1}=v$, then $v=t$ (and in that case $x_{t, u}=x_{t, w}$ ).

Using the property (2) stated above, we obtain the following:

THEOREM 2.1. If $x=x_{1} x_{2} \cdots x_{m}$ and $x=x_{1}^{\prime} x_{2}^{\prime} \cdots x_{n}^{\prime}$ are main biproducts of $x$ in $\boldsymbol{T}_{B}$ with the same generating pair $\{t, u\}$, then $m=n$ and $x_{i}=x_{i}^{\prime}$, for $i=$ $1,2, \ldots, m$. Specially, any maximal biproduct of $x \in \boldsymbol{T}_{B}$, that is a main biproduct, is uniquely determined.

\section{A construction of canonical biassociative groupoids}

A groupoid $\boldsymbol{G}=(G, \cdot)$ is said to be biassociative $[\mathbf{1}]$ if and only if for any $a, b \in$ $G$ the subgroupoid $S$ of $\boldsymbol{G}$ generated by $\{a, b\}$, i.e., $S=\langle a, b\rangle$, is a subsemigroup of $\boldsymbol{G}$. The class of all biassociative groupoids will be denoted by Bass. This class is hereditary and closed under the formation of homomorphic images and direct products, i.e., Bass is a variety of groupoids.

Assuming that $B$ is a nonempty set and $\boldsymbol{T}_{B}=\left(T_{B}, \cdot\right)$ the absolutely free groupoid with the free basis $B$, we are looking for a canonical groupoid in Bass, i.e., a groupoid $\boldsymbol{R}=(R, *)$ with the following properties:

i) $B \subset R \subset T_{B}$; ii) $t u \in R \Rightarrow t, u \in R$; iii) $t u \in R \Rightarrow t * u=t u$

iv) $\boldsymbol{R}$ is a free groupoid in Bass with the free basis $B$.

A "candidate" for the carrier $R$ of the desired groupoid $\boldsymbol{R}$ is the set defined by:

$$
R=\left\{x \in T_{B} \text { : every biproduct of any subterm of } x \text { is a main biproduct }\right\} \text {. }
$$

The following properties of $R$ are obvious corollaries of (3.1).

Proposition 3.1. a) $R$ satisfies i) and ii).

b) $x, y \in R \Rightarrow\{x y \notin R \Leftrightarrow x y$ has a biproduct that is not a main

c) $x, y \in T_{B} \Rightarrow\{x y \in R \Leftrightarrow x, y \in R \&$ every biproduct of any subterm biproduct in $\left.\boldsymbol{T}_{B}\right\}$ of xy in $\boldsymbol{T}_{B}$ is a main biproduct $\}$.

Lemma 3.1. For any $x \in R$ there is a unique maximal biproduct of $x$ in $\boldsymbol{T}_{B}$ that is a main biproduct.

Proof. Existence. By Proposition 2.2, any $x \in T_{B}$ has maximal biproducts in $\boldsymbol{T}_{B}$ and thus any $x \in R$ has maximal biproducts in $\boldsymbol{T}_{B}$. By the definition of $R$, every biproduct of any subterm of $x$ is a main biproduct and therefore the maximal biproducts of $x$ are main biproducts, too.

Uniqueness. Let $x \in R$ and $x_{\langle t, u\rangle}, x_{\langle v, w\rangle}$ be maximal biproducts of $x$ in $\boldsymbol{T}_{B}$. Since $x \in R$, both maximal biproducts $x_{\langle t, u\rangle}, x_{\langle v, w\rangle}$ are main biproducts and we will denote them by $x_{t, u}, x_{v, w}$. Let $x=x_{1} x_{2} \cdots x_{m}$ and $x=x_{1}^{\prime} x_{2}^{\prime} \cdots x_{m}^{\prime} x_{m+1}^{\prime} \cdots x_{m+k}^{\prime}$, $k \geqslant 0$, be the representations of $x$ as main biproducts in $\langle t, u\rangle$ and $\langle v, w\rangle$, respectively. By the property (2) we have that

$$
m-1=\chi_{\langle t, u\rangle}\left(x_{1} x_{2} \cdots x_{m}\right)=\chi_{\langle v, w\rangle}\left(x_{1}^{\prime} x_{2}^{\prime} \cdots x_{m+k}^{\prime}\right)=m+k-1,
$$


which implies that $k=0$ and that $x_{i}=x_{i}^{\prime}$, for $1 \leqslant i \leqslant m$. Therefore, the maximal biproducts $x_{t, u}$ and $x_{v, w}$ are in fact the same biproduct.

Bellow, for $x \in R$, we will denote by $x=x_{1} x_{2} \cdots x_{m}$ the maximal main biproduct of $x$ in $\boldsymbol{T}_{B}$ (if it is not stated otherwise).

LEMma 3.2. Let $x \in R$, let the maximal biproduct of $x$ be generated by $\{t, u\}$, and let another biproduct of $x$ be generated by $\{v, w\}$. Then $v, w \in\langle t, u\rangle$.

Proof. Let $x=x_{1} \cdots x_{m}$ be the maximal biproduct of $x$ generated by $\{t, u\}$ and let $x=x_{1}^{\prime} \cdots x_{n}^{\prime}$ be another biproduct of $x$ generated by $\{v, w\}$. By Proposition 2.3 we may put $t=v$. Both biproducts are equal and since $x \in R$, they are main biproducts. By Lemma 3.1, $n<m$, i.e., $m=n+k, k \geqslant 1$, so

$$
x_{1}^{\prime} \cdots x_{n}^{\prime}=x_{1} \cdots x_{n} x_{n+1} \cdots x_{n+k} .
$$

Using this facts, we obtain that $x_{i}^{\prime}=x_{i}=t$, for $i \in\{1, \ldots, n-1\}$. Clearly, $x_{n}^{\prime}=w$ and $x_{i} \in\{t, u\}$, for $i \in\{n, \ldots, n+k\}$. Therefore, $v, w \in\langle t, u\rangle$.

Proposition 3.2. Let $x, y \in R$ and the maximal biproducts $x=x_{1} x_{2} \cdots x_{m}$, $y=y_{1} y_{2} \cdots y_{n}$ have generating pairs $\{t, u\},\{v, w\}$, respectively. Then $x y \in R$ if and only if (a) or (b), where

(a) $y \notin\langle t, u\rangle$, and for any biproduct of $x$ with a generating pair $\left\{t_{1}, u_{1}\right\}$, if $t_{1}, u_{1} \in\langle v, w\rangle$, then $t_{1}=u_{1}=x$

(b) $y \in\langle t, u\rangle$ and $t=u=x \in B$.

Proof. Let $x y \in R$. There are two possible cases for $y: 1) y \notin\langle t, u\rangle$ and 2) $y \in\langle t, u\rangle$.

Case 1). Since $\{v, w\}$ is the generating pair for the biproduct $y=y_{1} y_{2} \cdots y_{n}$, and $y \notin\langle t, u\rangle$, we should consider the cases when some of the biproducts of $x$ has a generating pair $\left\{t_{1}, u_{1}\right\}$, such that $t_{1}, u_{1} \in\langle v, w\rangle$. Let $x=z_{1} z_{2} \cdots z_{k}$ be such a biproduct of $x$. Then $z_{i} \in\left\{t_{1}, u_{1}\right\} \subseteq\langle v, w\rangle$. The product $x y=$ $\left(z_{1} z_{2} \cdots z_{k}\right)\left(y_{1} y_{2} \cdots y_{n}\right)$ will be a main biproduct only if $k=1$, i.e., $x=z_{1}$, and $z_{1}=v$ or $z_{1}=w$. Since $x=z_{1}$ is generated by $\left\{t_{1}, u_{1}\right\}$, it follows that $t_{1}=u_{1}=x$.

Case 2). In this case $x y$ has a biproduct with a generating pair $\{t, u\} . x y$ is a main biproduct, since $x y \in R$ and, therefore $m=1$, i.e., $x=x_{1}$. The maximal biproduct of $x$ is generated by $\{t, u\}$, so $t=u=x$. Moreover, $x \in B$, because if $x \notin B$ (for example $x=a b$, i.e., $t=u=a b$ ), then the biproduct of $x y$ generated by $\{a, b\}$ can not be a main biproduct, that contradicts the assumption that $x y \in R$.

For the converse, let (a) or (b) hold. If (b) holds, then it is clear that $x y \in R$.

Let (a) holds and suppose $x y \notin R$. From 1 ) we obtain that $x \in\langle v, w\rangle$. Therefore, there is a biproduct of $x$ with a generating pair $\{v, w\}$. By Lemma 3.2 it follows that $v, w \in\langle t, u\rangle$, that contradicts the assumption that $y \notin\langle t, u\rangle$.

Now we define an operation $*$ on $R$ as follows. Let $x, y \in R, x=x_{1} x_{2} \cdots x_{m}$, $y=y_{1} y_{2} \cdots y_{n}$ and put

$$
x * y= \begin{cases}x y, & \text { if } x y \in R \\ x_{1} x_{2} \cdots x_{m} y_{1} y_{2} \cdots y_{n}, & \text { if } x y \notin R .\end{cases}
$$


The operation $*$ is well-defined, i.e., $\boldsymbol{R}=(R, *)$ is a groupoid. Namely, let $x, y \in$ $R$. If $x y \in R$, then $x * y$ is a uniquelly determined element of $R$. If $x y \notin R$, then $z=x_{1} x_{2} \cdots x_{m} y_{1} y_{2} \cdots y_{n}$ is a term of $T_{B}$ that is a main biproduct. Clearly, every biproduct of any subterm of $x_{1} x_{2} \cdots x_{m} y_{1} y_{2} \cdots y_{n}$ is a main biproduct. Therefore, by (3.1), $z \in R$. Since $x_{1} x_{2} \cdots x_{m} y_{1} y_{2} \cdots y_{n}$ as a maximal biproduct in $\boldsymbol{T}_{B}$ is unique (by Lemma 3.1), it follows that $x * y$ is uniquely determined element of $R$ in the case $x y \notin R$. Thus, $\boldsymbol{R}=(R, *)$ is a groupoid.

By (3.2) it follows directly that:

$1^{\circ}$. If $x y \in R$, then $x, y \in R \& x * y=x y$ (i.e., $\boldsymbol{R}$ satisfies ii) and iii)).

$2^{\circ} .(\forall x, y \in R)|x * y|=|x|+|y|$.

The following three properties of $\boldsymbol{R}\left(3^{\circ}-5^{\circ}\right)$ show that the groupoid $\boldsymbol{R}=(R, *)$ is free in Bass with the free basis $B$.

\section{$3^{\circ} . \boldsymbol{R} \in$ Bass.}

Proof OF $3^{\circ}$. We have to show that every subgroupoid of $\boldsymbol{R}$ generated by two elements is a subsemigroup of $\boldsymbol{R}$.

For this purpose, let $t, u \in R$ and $\langle t, u\rangle_{*}$ be the subgroupoid of $\boldsymbol{R}$ generated by $\{t, u\}$. According to the definition of $*$, any $x \in\langle t, u\rangle_{*}$ is a maximal biproduct with the generating pair $\{t, u\}$. Therefore, if $x, y, z \in\langle t, u\rangle_{*}$, then $x=x_{1} x_{2} \cdots x_{m}$, $y=y_{1} y_{2} \cdots y_{n}, z=z_{1} z_{2} \cdots z_{p}\left(x_{i}, y_{j}, z_{k} \in\{t, u\}\right)$ and by (3.2):

$$
(x * y) * z=x_{1} x_{2} \cdots x_{m} y_{1} y_{2} \cdots y_{n} z_{1} z_{2} \cdots z_{p}=x *(y * z),
$$

i.e., the subgroupoid $\langle t, u\rangle_{*}$ is a subsemigroup of $\boldsymbol{R}$. Hence, $\boldsymbol{R} \in$ Bass.

$4^{\circ}$. The set of primes in $\boldsymbol{R}$ coincides with $B$ and generates $\boldsymbol{R}$.

(An element $a$ in a groupoid $\boldsymbol{G}=(G, \cdot)$ is said to be prime in $\boldsymbol{G}$ if and only if $a \neq x y$, for any $x, y \in G$.)

Proof OF $4^{\circ}$. If $b \in B$, then by $(3.2) b \neq x * y$, for all $x, y \in R$. Hence, every $b \in B$ is prime in $\boldsymbol{R}$. To show that no element of $R \backslash B$ is prime in $\boldsymbol{R}$, let $x \in T_{B} \backslash B$ be a term belonging to $R$. Then by (3.1), every biproduct of any subterm of $x$ is a main biproduct, and thus the maximal biproduct of $x$ in $\boldsymbol{T}_{B}$ is a main biproduct. Therefore, $x=x_{1} x_{2} \cdots x_{m}$, where $m \geqslant 2$ (since $x \in T_{B} \backslash B$ ). Thus, $x=x_{1} *\left(x_{2} \cdots x_{m}\right)$, i.e., $x$ is not prime in $\boldsymbol{R}$.

Let $\boldsymbol{Q}$ be the subgroupoid of $\boldsymbol{R}$ generated by $B, \boldsymbol{Q}=\langle B\rangle_{*}$. We will show that $R=Q$. Clearly, $Q \subseteq R$. To show that $R \subseteq Q$, let $x \in R$. If $x \in B$, then $x \in\langle B\rangle_{*}=Q$, i.e., $(x \in R \&|x|=1 \Rightarrow x \in Q)$.

Suppose that $(x \in R \&|x| \leqslant k \Rightarrow x \in Q)$ is true. If $x \in R$ is such that $|x|=$ $k+1$, then $x=x_{1} x_{2}$ in $\boldsymbol{T}_{B}$ and $\left|x_{1}\right|,\left|x_{2}\right| \leqslant k$. By the inductive hypothesis we have $x_{1}, x_{2} \in Q$, and since $\boldsymbol{Q}$ is a groupoid, it follows that $x=x_{1} x_{2}=x_{1} * x_{2} \in Q$. Thus, $R \subseteq Q$. Therefore, $\boldsymbol{R}=\boldsymbol{Q}=\langle B\rangle_{*}$.

$5^{\circ}$. If $\boldsymbol{G} \in$ Bass and $\lambda: B \rightarrow G$ is a mapping, then there is a homomorphism $\psi: \boldsymbol{R} \rightarrow \boldsymbol{G}$ that extends $\lambda$, i.e., $\psi(b)=\lambda(b)$, for all $b \in B$.

Proof of $5^{\circ}$. Let $\varphi: \boldsymbol{T}_{B} \rightarrow \boldsymbol{G}$ be the homomorphism that extends $\lambda$. Denote by $\psi$ the restriction of $\varphi$ on $R$ (i.e., $\psi=\left.\varphi\right|_{R}$ ). It suffices to show that

$$
(\forall x, y \in R) \varphi(x * y)=\varphi(x) \varphi(y) .
$$


Let $x, y \in R$. If $x y \in R$, then $\varphi(x * y)=\varphi(x y)=\varphi(x) \varphi(y)$. If $x y \notin R$ (i.e., $x=x_{1} x_{2} \cdots x_{m}, y=y_{1} y_{2} \cdots y_{n}$, where $x_{i}, y_{j} \in\{t, u\}$ and $\left.m \geqslant 2\right)$ then using the fact: $\left(x_{i}, y_{j} \in\{t, u\} \Rightarrow \varphi\left(x_{i}\right), \varphi\left(y_{j}\right) \in\{\varphi(t), \varphi(u)\}\right)$ we have

$$
\begin{aligned}
\varphi(x * y) & =\varphi\left(x_{1} \cdots x_{m} y_{1} \cdots y_{n}\right)=\varphi\left(x_{1}\right) \cdots \varphi\left(x_{m}\right) \varphi\left(y_{1}\right) \cdots \varphi\left(y_{n}\right) \\
& =[\boldsymbol{G} \in \mathbf{B a s s}]=\varphi\left(x_{1} \cdots x_{m}\right) \varphi\left(y_{1} \cdots y_{n}\right)=\varphi(x) \varphi(y) .
\end{aligned}
$$

So, the conditions i)-iv) at the beginning of this section are fulfilled and thus we proved the following

TheOREM 3.1. The groupoid $\boldsymbol{R}=(R, *)$, defined by (3.1) and (3.2) is a canonical biassociative groupoid with a free basis $B$.

\section{Acknowledgements}

This work is partly supported by Macedonian Academy of Sciences and Arts within the project "Algebraic structures". Authors would like to express their sincere gratitude to Professor G. Čupona, the project manager, and special thanks to the referee for the valuable comments and suggestions.

\section{References}

[1] S. Ilić, B. Janeva, N. Celakoski, Free Biassociative groupoids, Novi Sad J. Math. 35:1 (2005), $15-23$

[2] R. N. McKenzie, G. F. McNulty, W. F. Taylor, Algebras, Latices, Varieties, Vol. I, Wadsworth and Brooks/Cole, Monterey, 1987

[3] G. Čupona, N. Celakoski, S. Ilić, On Monoassociative groupoids, Mat. Bilten 26 (2002), 5-16

[4] G. Cupona, N. Celakoski, B. Janeva, Canonical groupoids with $x^{m} \cdot y^{n}=x y$, Mat. Bilten 23 (1999), 11-18

Prirodno-matematički fakultet

Skopje, p.f. 162

Macedonia

(B. Janeva, V. Celakoska-Jordanova)

biljana@ii.edu.mk

vesnacj@iunona.pmf.ukim.edu.mk

Prirodno-matematički fakultet, 18000 Niš, Serbia

sneska@pmf .ni.ac.yu 